Artículo / Investigación

Article / Research

\section{Malnutrición y pobreza estructural. Comparación de dos cohortes de escolares de Puerto Madryn, Argentina}

\author{
Malnutrition and structural poverty. Comparison of two schoolchildren \\ cohorts from Puerto Madryn, Argentina
}

\author{
Bárbara Navazo, Silvia L. Dahinten y Evelia E. Oyhenart
}

Recibido 30 octubre 2017 / Enviado para modificación 2 noviembre 2017 / Aceptado 28 diciembre 2017

\section{RESUMEN}

BN: Lic. Antropología. Igevet, Facultad de Ciencias Veterinarias, UnLP-CCT La Plata, coNicet. Facultad de Ciencias Naturales y Museo, Universidad Nacional de La Plata. La Plata, Buenos Aires, Argentina.

bnavazo@fcnym.unlp.edu.ar

SD: Lic. Antropología. Ph. D. Ciencias Naturales. ideAus-Conicet. Facultad de Ciencias Naturales San Juan Bosco, Universidad Nacional de la Patagonia. Puerto Madryn. Chubut, Argentina.dahinten@cenpat-conicet.gob.ar EO: Lic. Antropología. Ph. D. Ciencias Naturales. Igevet, Facultad de Ciencias Veterinarias, Unlp-Cct La Plata, conicet. LinoA, Facultad de Ciencias Naturales y Museo, Universidad Nacional de La Plata. Buenos Aires, Argentina. oyhenart@fcnym.unlp.edu.ar

Objetivo Estimar el estado nutricional de dos cohortes (C) de niños concurrentes a escuelas de barrios con diferentes Necesidades Básicas Insatisfechas (NBI) de Puerto Madryn, Argentina.

Métodos Investigación transversal. Se midieron peso y talla de niños de seis a 11 años asistentes a escuelas ubicadas en siete barrios. La $C 1 \quad(n=2$ 040) fue relevada entre 2001-2006 y C2 ( $n=1$ 696) entre 2014-2016. Se calculó el Índice de Masa Corporal y se emplearon los puntos de corte de la OMS para determinar el estado nutricional. Para estimar la pobreza estructural se extrajeron, para 2001 y 2010 , los porcentajes de $\mathrm{NBI}$ censales de cada barrio. Las prevalencias de estado nutricional entre cohortes y categorías de $\mathrm{NBI}$ se compararon mediante $\mathrm{Chi}^{2}$.

Resultados En los siete barrios analizados, las NBI fueron: $0 \%-10 \%(n=3), 10 \%-$ $25 \%(n=2)$ y $25 \%-50 \%(n=1)$, sin variación entre $2001-2010$. En otro barrio, las $\mathrm{NBI}$ aumentaron de $25 \%-50 \%$ a $>50 \%$. Al evaluar el estado nutricional de los niños de cada escuela hubo diferencias para obesidad en la mayoría de ellas y para baja talla/edad y sobrepeso solo en una. La comparación C1-C2 indicó diferencias para obesidad.

Conclusiones Los barrios de Puerto Madryn conservan, en gran parte, sus niveles de pobreza estructural. No ocurre lo mismo con el estado nutricional de los niños: mientras las prevalencias de desnutrición crónica se mantienen o descienden, las de obesidad aumentan en todos los barrios. El análisis efectuado permite evaluar transformaciones del contexto de residencia de la población e identificar grupos vulnerables.

Palabras Clave: Desnutrición; sobrepeso; obesidad (fuente: DeCS, BIREME).

\section{ABSTRACT}

Objective To assess nutritional condition in two cohorts (C) of children from households with different Unsatisfied Basic Needs (UBN) attending neighborhood schools in Puerto Madryn, Argentina.

Methods A cross-sectional study on six to 11 year-old children attending seven neighborhood schools surveyed in the periods 2001-2006 (C1, n=2 040) and 2014-2016 (C2, $\mathrm{n}=1696$ ) was performed. Weight, height and body mass index were measured using the World Health Organization cut-off values for nutritional condition. Neighborhood structural poverty was determined with percent UBN from the 2001 and 2010 census. Chi ${ }^{2}$ was used to compare the prevalence of nutritional condition between $\mathrm{C}$ and UBN categories. Results In the seven neighborhoods analyzed, percent UBN was $0 \%-10 \%(n=3)$, $10 \%-25 \%(n=2)$ and $25 \%-50 \%(n=1)$, without changes in the period $2001-2010$. In the remaining neighborhood, UBN increased from $25 \%-50 \%$ to $>50 \%$. The nutritional evaluation of children showed significant differences in the prevalence of obesity in most schools, while low height-for-age and overweight prevalence was significant only in one school. Comparison of $\mathrm{C} 1$ and $\mathrm{C} 2$ only showed significant differences in the prevalence of obesity. 
Conclusions Structural poverty rates in Puerto Madryn neighborhoods were mostly unchanged; however, the nutritional condition of children was different: while the prevalence of chronic malnutrition did not change or decreased, the prevalence of obesity increased in all neighborhoods. Our findings could help to determine changes in the context of resident population and identify vulnerable groups.

Key Words: Undernutrition; overweight; obesity (source: MeSH, NLM).

$\mathrm{L}$ a ciudad de Puerto Madryn (Chubut) ha experimentado uno de los mayores crecimientos demográficos de la República Argentina. Dicho proceso se inició en la década de 1970 con la puesta en funcionamiento de la empresa productora de aluminio primario (ALUAR), el desarrollo de la industria pesquera en la década siguiente, conjuntamente con el incremento del turismo y de los servicios vinculados en la década de 1990 (1). Como consecuencia de ello, en el período 1970-2010 la ciudad incrementó su población pasando de 6100 habitantes a alrededor de 80 ooo, según los datos del Censo Nacional de Población, Hogares y Viviendas (2). La población migrante provino de provincias argentinas tales como Mendoza, Río Negro y Buenos Aires, así como de países limítrofes (Bolivia y Chile, entre otros) (3).

Este incremento poblacional tuvo efectos sobre el tejido urbano que se expandió en forma no planificada y sin que fuera acompañado por políticas habitacionales gubernamentales, por lo que el acceso a la tierra y a la vivienda se convirtió en un serio problema a resolver $(4,5)$. Una gran mayoría de los migrantes tendió a localizarse en los márgenes oeste y norte de la ciudad, en las zonas más baratas y menos apetecibles para los mercados inmobiliario y turístico (4). Los barrios y los asentamientos de estas áreas presentaron indicadores sociales de alta vulnerabilidad sanitaria y socioeconómica como consecuencia de la carencia en los servicios (electricidad, agua potable, gas, salud), en la mensura de los terrenos y en el transporte público (6). Los barrios San Miguel, Agustín Pujol I y II, entre otros, correspondieron a estas zonas y contaban con elevado porcentaje de hogares con Necesidades Básicas Insatisfechas (N BI) (7).

El método NBI es una herramienta creada a fines de los años 70 con el objeto de medir y/o caracterizar la pobreza, mediante un enfoque directo, aprovechando la información censal. Es así que, a partir de los censos de población y vivienda, se verifica si los hogares satisfacen o no una serie de indicadores elementales que permiten a las personas tener una vida digna de acuerdo con las normas sociales vigentes (8). Las NBI se relacionan con la pobreza «de largo plazo» o «estructural» puesto que los indicadores utilizados no son sensibles a las fluctuaciones económicas o sociales de corto o mediano plazo (9). De este modo, cuando los hogares o la población que vive en los mismos, no logran satisfacer tales necesidades son categorizados con NBI (10). El Instituto Nacional de Estadísticas y Censos (8) considera que un hogar es pobre por NBI si presenta al menos una de las siguientes carencias: a) Hacinamiento: más de tres personas por cuarto; b) Vivienda inadecuada: pieza de inquilinato, vivienda precaria u otro tipo, lo que excluye casa, departamento y rancho; c) Condiciones sanitarias: falta de retrete; d) Menores no escolarizados: al menos un niño de seis a 12 años que no asiste a la escuela; y e) Capacidad de subsistencia: cuatro o más personas por miembro ocupado y jefe de hogar que no haya completado el tercer grado de escolaridad primaria. Para seleccionar estos indicadores, en América Latina se ha utilizado un método denominado de realización combinada: un hogar se considera pobre si no alcanza el umbral de al menos un indicador. Esta idea parte de dos premisas: 1) todas las necesidades son básicas -es decir, fundamentales para reflejar la pobreza- y, por lo tanto, 2) todas tienen la misma importancia. Es por ello que un hogar es clasificado como pobre cuando no logra satisfacer al menos una de las necesidades básicas (11).

En Argentina, la metodología de las NBI fue diseñada con el objetivo de construir mapas de pobreza que permitieran identificar en la forma más desagregada posible las carencias críticas que predominaban en cada una de las regiones del país y elaborar políticas focalizadas (12). De acuerdo con Montilva (13) es deseable establecer la relación entre nutrición y pobreza en localidades pequeñas, tales como los barrios a nivel urbano, donde la información obtenida puede ser de gran utilidad para planificar, priorizar, implantar, monitorear y evaluar los efectos de acciones focalizadas dirigidas a los grupos más necesitados. En este sentido, el estudio del crecimiento físico de los niños y los jóvenes es considerado un indicador sensible de la calidad del entorno social, económico y político en el que viven $(14,15)$. Con relación a ello, la antropometría ha sido empleada amplia y exitosamente en la evaluación del riesgo de salud y nutricional, especialmente de niños, siendo útil para la investigación de la historia económica (16); sin embargo, los indicadores antropométricos no pueden ser sustitutos, sino son complementarios de las medidas convencionales de los estándares de vida, ampliando el poder del análisis histórico-económico (17). Es por ello que, frecuentemente se emplean variables an- 
tropométricas como peso corporal y talla vinculadas con el método de las NBI (1 8-21). Lo expuesto anteriormente motivó la realización del presente trabajo.

El objetivo fue estimar el estado nutricional, transcurridos diez años, de dos cohortes de niños concurrentes a escuelas ubicadas en barrios con diferentes Necesidades Básicas Insatisfechas, localizados en Puerto Madryn (Chubut, Argentina).

\section{MÉTODOS}

\section{Relevamiento antropométrico}

El estudio realizado fue de corte transversal. Se emplearon técnicas antropométricas inocuas que no afectaron la integridad física, psíquica y moral de los niños, respetando el artículo Iv del Código de Nüremberg y de acuerdo a normas internacionales estandarizadas (22). Fueron excluidos del estudio tanto los niños con enfermedad manifiesta y/o indicación medicamentosa al momento del relevamiento (según constancia obrante en los registros institucionales), como aquellos que no contaban con autorización escrita de sus padres/tutores y los que aun teniéndola, manifestaron negativa a ser medidos.

La recolección de los datos antropométricos se realizó en los términos de la Ley 25326 (art. 4, 5 y 6). Todos los datos personales, particularmente los sensibles en términos del artículo $2^{\circ}$, fueron sometidos a procedimientos de disociación. La transferencia de resultados se efectuó preservando la identidad del niño (Ley 26 343, art. 11 y 12).

La muestra incluyó dos cohortes (C) de niños:

Cohorte 1 ( $\left.\mathrm{C}_{1}\right)$ : El estudio fue realizado, entre los años 2001 y 2006, en escolares concurrentes a establecimientos educativos ubicados en los barrios: Agustín Pujol I y II, Del Desembarco; Gobernador Fontana, Julio Roca, Parry Madryn, y San Miguel, en el marco del proyecto PROBIEDMA $(23,24)$. La muestra quedó conformada por 2040 niños (varones $=51,3 \%$ y mujeres $=48,6 \%$ ), de seis a 11 años de edad (Tabla 1 ).

Cohorte 2 ( $\left.\mathrm{C}_{2}\right)$ : El estudio fue llevado a cabo entre los años 2014 y 2016, en los mismos establecimientos educativos de la ciudad de Puerto Madryn que habían sido relevados entre 2001 y $2006\left(\mathrm{C}_{1}\right)$. La muestra quedó conformada por 1696 escolares (varones $=46,6 \%$ y mujeres $=53,3 \%$ ) de seis a 11 años de edad (Tabla 1 ).

Tabla 1. Composición de la muestra

\begin{tabular}{|c|c|c|}
\hline Escuela & $\mathrm{C} 1$ & $\mathrm{C} 2$ \\
\hline Provincial N 49 & 538 & 324 \\
\hline Provincial No 84 & 321 & 363 \\
\hline Provincial Nº 158 & 394 & 226 \\
\hline Provincial Nº 213 & 196 & 143 \\
\hline Provincial Nº 219 & 193 & 304 \\
\hline Municipal $N^{\circ} 3$ & 221 & 216 \\
\hline Municipal No 1 & 177 & 120 \\
\hline Total & 2040 & 1696 \\
\hline
\end{tabular}

La metodología utilizada fue la misma en ambas cohortes. Se relevaron las variables: a) peso (P), en kilogramos, con balanza digital portátil (100 g de precisión). En todos los casos los niños vistieron ropa liviana cuyo peso se descontó del peso total del niño y b) talla ( $\mathrm{T})$, en centímetros, utilizando un antropómetro vertical (1 mm de precisión).

Se calculó el Índice de Masa Corporal $\left(\mathrm{IMC}=\mathrm{P} / \mathrm{T}^{2}\right)$, se determinaron los indicadores nutricionales: Bajo IMC (BIMC), Baja Talla/Edad (B T/E), Sobrepeso (s) y Obesidad (o), empleando los puntos de corte recomendados por la Organización Mundial de la Salud (OMS) (BIMC $<-2$; BT/ $\mathrm{E}<-2 ; \mathrm{S}>1 \mathrm{y} \leq 2 ; \mathrm{O}>2$ ) y se calcularon sus prevalencias.

\section{Zona de estudio}

Cada escuela fue referenciada al barrio en donde se emplazaba (Tabla 2; Figura 1). De acuerdo con Sassone (5) la estructura urbana de Puerto Madryn está conformada por tres sectores diferenciados, pudiéndose plantear que tres ciudades conviven en una: a) la ciudad portuario-industrial; b) la ciudad ventana al mundo; y c) la ciudad popular.

"La ciudad portuario-industrial» coincide con el Distrito Norte y el Distrito Industrial. El primero incluye al barrio Julio Argentino Roca (Escuela Provincial $\mathrm{N}^{\mathrm{O}} 49$ ), construido por la empresa ALUAR para otorgar viviendas en propiedad a sus empleados. Por su parte, el Distrito Industrial carece de áreas residenciales y cuenta con cinco parques industriales.

Tabla 2. Ubicación geográfica de las escuelas e Índice de Necesidades Básicas Insatisfechas (NBI) de las familias

\begin{tabular}{|c|c|c|c|c|}
\hline Escuela & Barrio & $\begin{array}{l}\text { Ubicación Geográfica } \\
\text { (Distrito) }\end{array}$ & NBI 2001 (\%) & NBI 2010 (\%) \\
\hline Provincial $N^{\circ} 49$ & Julio Roca & Norte & $0-10$ & 0 -a 10 \\
\hline Provincial Nº 84 & Parry Madryn & Centro & $0-10$ & $0-10$ \\
\hline Provincial Nº 158 & Del Desembarco & Sur & $0-10$ & $0-10$ \\
\hline Provincial Nº 213 & Gobernador Fontana & Oeste & $10-25$ & $10-25$ \\
\hline Municipal $N^{\circ} 1$ & Agustín Pujol I & Noroeste & $10-25$ & $10-25$ \\
\hline Municipal $N^{\circ} 3$ & San Miguel & Oeste & $25-50$ & $25-50$ \\
\hline Provincial Nº 219 & Agustín Pujol II & Noroeste & $25-50$ & $>50$ \\
\hline
\end{tabular}




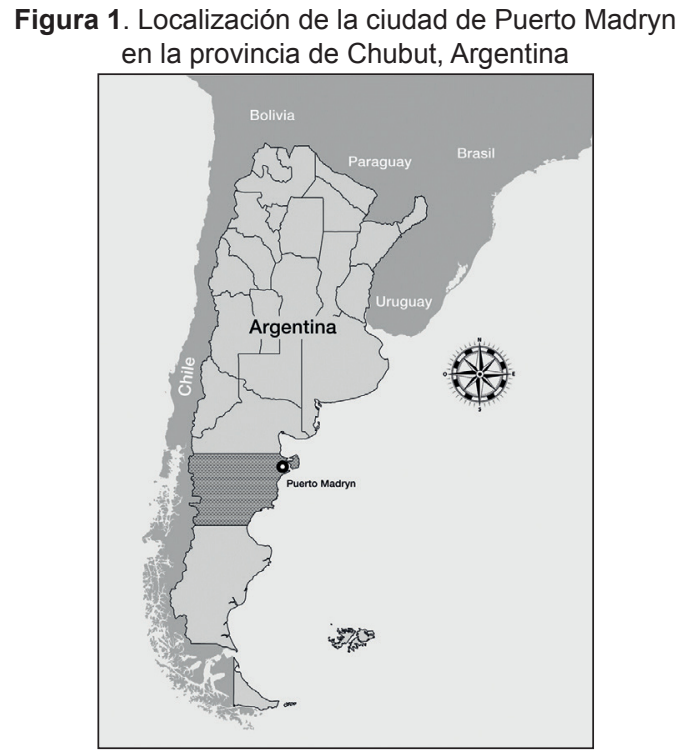

«La ciudad ventana al mundo» ubicada hacia el Este, sobre el litoral del Golfo Nuevo, incluye el centro comercial bancario y de servicios, viviendas residenciales, condominios, hoteles cinco estrellas y un polo científico. Se corresponde con los Distritos Centro y Sur. El primero comprende barrios como el Parry Madryn (Escuela Provincial $\mathrm{N}^{\circ} 84$ ), mientras que el segundo al Del Desembarco (Escuela Provincial No 158 ).

Al Oeste se extiende "la ciudad popular", modelo de ciudad fragmentada, como otras de la Patagonia (San Carlos de Bariloche o Comodoro Rivadavia), donde el componente migratorio, como vector de la diversidad cultural, es eje explicativo también de la fragmentación espacial. Esta zona comprende los barrios más alejados del litoral surgidos a mediados de los ochenta, en los que luego de dos décadas de un importante crecimiento demográfico surgieron loteos sociales municipales y viviendas construidas con planes provinciales y nacionales, con calles de tierra, sin árboles, sin alcantarillas, anegables y de topografía difícil. A modo de ejemplo, se pueden mencionar los barrios de los Distrito Oeste: San Miguel (Escuela Municipal $\mathrm{N}^{\circ} 3$ ) y Gobernador Fontana (Escuela Provincial $\mathrm{N}^{\mathrm{O}}$ 213) y Noroeste: Agustín Pujol I (Escuela Municipal $\mathrm{N}^{\mathrm{O}} 1$ ) y Agustín Pujol II (Escuela Provincial $\mathrm{N}^{\mathrm{O}} 219$ ).

A partir de la revisión de los registros escolares, se constató que alrededor del $80 \%$ de los niños residía en el mismo barrio donde se encontraba emplazada la escuela a la que asistían y el $20 \%$ restante, en las cercanías.

\section{Metodología de las NBI}

Para poder aplicar esta metodología se relevaron los porcentajes de NBI para cada barrio de acuerdo con los datos censales de los años 2001 y 2010 , que fueran publicados por la Dirección General de Estadísticas y Censos de Chubut (7). A pesar de que en el empleo de las NBI, no todos los indicadores pueden aplicarse a la totalidad de los hogares, se decidió utilizarlas debido a que su implementación conjunta con el análisis del estado nutricional resulta de utilidad para identificar la población vulnerable, permitiendo a su vez evaluar las transformaciones de su contexto de residencia (25).

Se empleó el test de $\mathrm{Chi}^{2}$ para comparar las prevalencias para cada estado nutricional de $\mathrm{C}_{1}$ y $\mathrm{C}_{2}$ en las categorías de NBI como así también en cada barrio.

\section{RESULTADOS}

Los barrios Julio Roca, Parry Madryn y Del Desembarco se incluyeron en la categoría de NBI O\%-10\%, manteniendo dichos valores en los dos momentos considerados. Los valores de NBI fueron mayores en los barrios Gobernador Fontana y Agustín Pujol I (10\%-25\%) y San Miguel (25\%-50\%) y no mostraron variación entre los años 2001 y 2010. Por último, en el barrio Agustín Pujol II el porcentaje de NBI fue de $25 \%-50 \%$ en el año 2001 a $>50 \%$ en el año 2010 (Tabla 2, Fig. 2).La comparación del estado nutricional de los niños indicó que para todas las categorías de NBI, sólo hubo diferencias significativas entre cohortes para obesidad (Tabla 3 ).

Por último, también se encontraron diferencias significativas en вт/в y Sobrepeso entre los niños de la $\mathrm{C}_{1}$ respecto de la $\mathrm{C}_{2}$ concurrentes a la escuela Municipal $\mathrm{N}^{\mathrm{O}}{ }_{1}$ (barrio Agustín Pujol I). Para la Obesidad en cambio, las diferencias correspondieron a los asistentes a las escuelas provinciales $\mathrm{N}^{\mathrm{O}} 49$ (barrio Julio Roca), $\mathrm{N}^{\mathrm{O}} 84$ (barrio Parry Madryn), N 213 (barrio Gobernador Fontana) y N ${ }^{\mathrm{O}} 219$ 
Figura 2. Ciudad de Puerto Madryn. Hogares con Necesidades Básicas Insatisfechas (NBI). Año 2001 Izquierda; año 2010 derecha

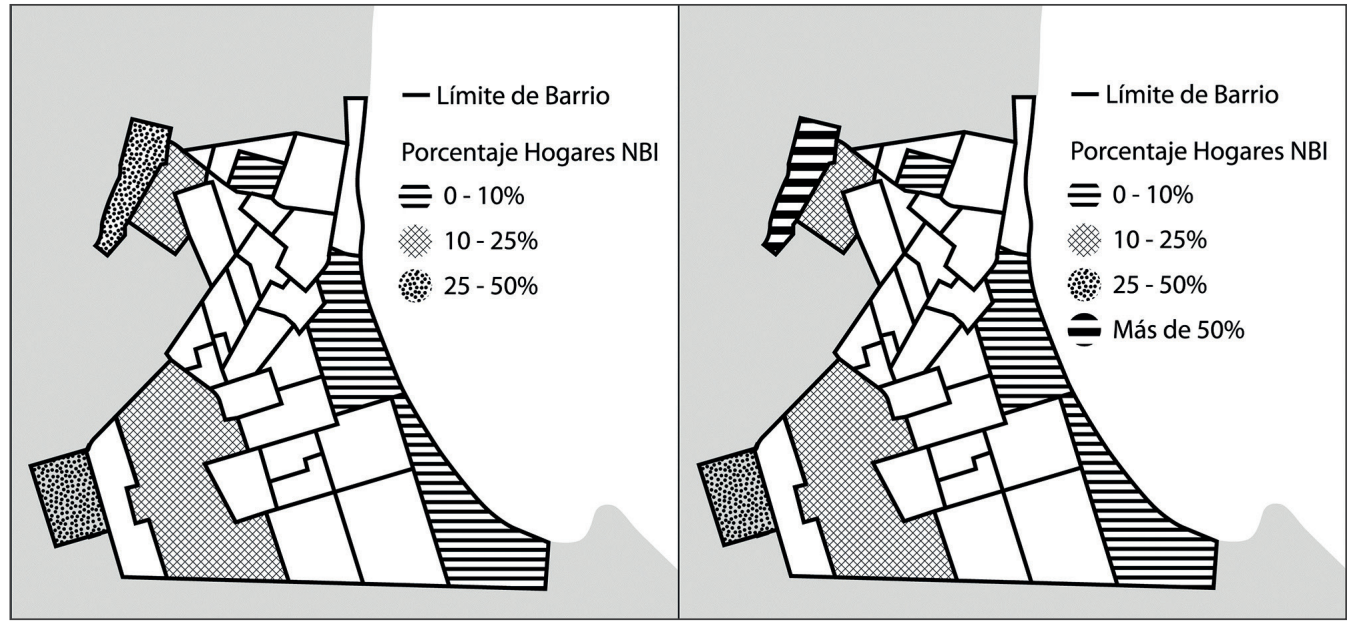

Tabla 3. Prevalencias de estado nutricional de los niños por categoría de Necesidades Básicas Insatisfechas (NBI)

\begin{tabular}{|c|c|c|c|c|c|c|c|c|c|c|c|c|c|c|c|c|}
\hline \multirow{2}{*}{ Indicador } & \multicolumn{4}{|c|}{ NBI $0-10 \%$} & \multicolumn{4}{|c|}{ NBI $10-25 \%$} & \multicolumn{4}{|c|}{ NBI $25-50 \%$} & \multicolumn{4}{|c|}{ NBI $25-50 \%$ a $\mathrm{NBI}>50 \%$} \\
\hline & C1 & $\mathrm{C} 2$ & $\mathrm{Chi}^{2}$ & $\mathrm{p}$ & C1 & $\mathrm{C} 2$ & $\mathrm{Chi}^{2}$ & $\mathrm{p}$ & C1 & $\mathrm{C} 2$ & $\mathrm{Chi}^{2}$ & $\mathrm{p}$ & C1 & $\mathrm{C} 2$ & $\mathrm{Chi}^{2}$ & $\mathrm{p}$ \\
\hline Bajo IMC & 0,7 & 0,4 & 0,695 & 0,295 & 1,1 & 0,0 & 2,838 & 0,118 & 0,5 & 0,5 & 0,000 & 0,745 & 0,0 & 0,3 & 0,636 & 0,612 \\
\hline Baja Talla/Edad & 1,4 & 1,3 & 0,058 & 0,482 & 5,9 & 3,4 & 2,040 & 0,106 & 4,1 & 1,4 & 2,946 & 0,076 & 4,1 & 4,3 & 0,005 & 0,569 \\
\hline Sobrepeso & 24,2 & 24,4 & 0,017 & 0,468 & 27,9 & 30,8 & 0,636 & 0,239 & 25,3 & 24,1 & 0,094 & 0,423 & 31,6 & 28,6 & 0,504 & 0,271 \\
\hline Obesidad & 14,2 & 22,6 & 25,290 & 0,000 & 12,6 & 20,5 & 7,264 & 0,005 & 5,9 & 24,5 & 29,647 & 0,000 & 12,4 & 24,7 & 11,080 & 0,001 \\
\hline
\end{tabular}

Tabla 4. Estado nutricional de los niños asistentes a las escuelas. Comparación entre cohortes

\begin{tabular}{|c|c|c|c|c|c|c|c|c|c|c|c|c|c|c|}
\hline \multirow{2}{*}{ Escuela } & \multirow{2}{*}{ Barrio } & \multirow{2}{*}{ NBI (\%) } & \multicolumn{4}{|c|}{ BAJO IMC (\%) } & \multicolumn{4}{|c|}{ BAJA TALLA/EDAD (\%) } & \multicolumn{4}{|c|}{ SOBREPESO (\%) } \\
\hline & & & C1 & $\mathrm{C} 2$ & $\mathrm{Chi}^{2}$ & $\mathrm{p}$ & C1 & $\mathrm{C} 2$ & $\mathrm{Chi}^{2}$ & $p$ & C1 & $\mathrm{C} 2$ & $\mathrm{Chi}^{2}$ & $\mathrm{p}$ \\
\hline Provincial $N^{\circ} 49$ & Julio Roca & $0-10$ & 0,2 & 0,6 & 1,085 & 0,317 & 1,3 & 1,5 & 0,086 & 0,492 & 27,1 & 26,2 & 0,084 & 0,418 \\
\hline Provincial $N^{0} 84$ & Parry Madryn & $0-10$ & 0,3 & 0,6 & 0,224 & 0,546 & 1,6 & 1,4 & 0,038 & 0,546 & 23,4 & 24,8 & 0,190 & 0,365 \\
\hline Provincial Nº 158 & Del Desembarco & $0-10$ & 1,8 & 0,0 & NC & - & 1,5 & 0,9 & 0,459 & 0,393 & 20,8 & 21,2 & 0,016 & 0,489 \\
\hline Provincial $N^{\circ} 213$ & $\begin{array}{l}\text { Gobernador } \\
\text { Fontana }\end{array}$ & $10-25$ & 1,5 & 0,0 & NC & - & 4,1 & 4,2 & 0,003 & 0,583 & 29,1 & 24,5 & 0,887 & 0,387 \\
\hline Municipal $N^{0} 1$ & Agustín Pujol I & $10-25$ & 0,6 & 0,0 & NC & - & 7,9 & 2,5 & 3,878 & 0,039 & 26,6 & 38,3 & 4,614 & 0,022 \\
\hline Municipal Nº3 & San Miguel & $25-50$ & 0,5 & 0,5 & 0,000 & 0,745 & 4,1 & 1,4 & 2,946 & 0,076 & 25,3 & 24,1 & 0,094 & 0,423 \\
\hline Provincial № 219 & Agustín Pujol II & $25-50 ;>50$ & 0,0 & 0,3 & NC & - & 4,1 & 4,3 & 0,005 & 0,569 & 31,6 & 28,6 & 0,504 & 0,271 \\
\hline
\end{tabular}

(barrio Agustín Pujol II); y las municipales $\mathrm{N}^{\mathrm{O}} 1$ (barrio Agustín Pujol I) y ${ }^{\circ} 3$ (barrio San Miguel) (Tabla 4 ).

\section{DISCUSIÓN}

En la mayoría de los barrios analizados los valores de las NBI no se modificaron en el período 2001-2010. La excepción fue el barrio Agustín Pujol i i en el cual los porcentajes de NBI cambiaron de 25\%-50\% a más de 50\%, indicando un aumento de la pobreza estructural en los hogares de las familias de la zona.

Diversos autores han planteado la importancia de analizar los barrios con relación a la salud de las personas que residen en ellos. El hecho de que un barrio no cuente con disponibilidad y accesibilidad a servicios sanitarios, alimentos sanos o que registre contaminación ambiental, pueden ser elementos nocivos para la salud de quienes habitan en él $(26,27)$. Según Kawachi y Berkman (28), distintos aspectos del entorno barrial han sido vinculados a factores tales como mortalidad infantil, bajo peso al nacer, tabaquismo y depresión. Sus condiciones representan entonces, contextos sociales y comunitarios dentro de los cuales pueden ocurrir cambios en los comportamientos individuales tales como la inactividad física, el sedentarismo y la mala alimentación $(28,29)$; la privación socioeconómica, las condiciones precarias de vivienda, y la falta de aceras, parques y áreas de recreo que se creen, conducen al aumento del riesgo de obesidad (30,31).

El análisis del estado nutricional de los niños de Puerto Madryn evidenció la coexistencia de ambos extremos de la malnutrición en los barrios. En el caso de la malnutrición por déficit, se observó que en el barrio Agustín Pujol 
I, la BT/E disminuyó en 5,4\%; sin embargo, en los restantes barrios las prevalencias se mantuvieron, aún en el barrio Pujol i en el cual la pobreza estructural aumentó. Es probable que la reducción de la desnutrición crónica encontrada en el Pujol I se relacione con la aplicación del Programa de Mejoramiento Barrial (PRomeBA). Entre los años 2005 y 2013 este programa benefició a más del 20\% de la población de Puerto Madryn y tuvo como finalidad consolidar los asentamientos informales a través de la legalización de la tenencia de la tierra, las obras de acceso a servicios públicos, las mejoras en las viviendas y entornos urbanos, y de este modo combatir en forma focalizada y territorial la pobreza. En particular, las obras realizadas en el barrio Pujol I incluyeron saneamiento, equipamiento urbano y comunitario, estabilización de suelos, alumbrado, cloacas y gas (32). Otro caso interesante fue lo observado en el barrio Agustín Pujol i en el cual los porcentajes de las NBI cambiaron de 25\%-50\% a más de $50 \%$. Dicho aumento pudo deberse, en parte, a que durante los primeros años del siglo Xxi este barrio fue uno de los sectores más excluidos de la ciudad. Cuando en el 2005 ALUAR realizó obras de ampliación y arribaron a la zona migrantes internos y externos como mano de obra, Pujol II se convirtió en uno de los sectores más populoso de Puerto Madryn. La escasez de viviendas y los costos elevados de los alquileres hicieron surgir asentamientos con indicadores sociales de alta vulnerabilidad sanitaria y socioeconómica (6). No obstante la situación descrita, la prevalencia de retardo linear de crecimiento de los niños se mantuvo en el orden del $4 \%$ a pesar de que, como lo expresara Pedraza (33), en este contexto era de esperar que la desnutrición se elevara con el incremento de la pobreza estructural. Posiblemente el hecho de que no se registrara incremento de la desnutrición pudo deberse a que diversas redes sociales contribuyeron a la contención y a la radicación en el barrio de los recién llegados (34).

En cuanto a la obesidad, la comparación entre las dos cohortes relevadas indicó que la mayor diferencia (18,6\%) se registró en el barrio San Miguel con 25\%-50\% de NBI, seguida por el 13,4\% encontrado en Parry Madryn, un barrio con o\%-10\% de NBI. Los incrementos más significativos de obesidad se registraron tanto en barrios con alto NBI como en aquellos con valores bajos. Esto podría relacionarse al planteo de Aguirre (35), quien propuso que habría dos tipos de niños «gordos». Por un lado estarían los de la opulencia, resultado del mayor consumo de "snacks», gaseosas y alto sedentarismo. Por el otro, los de la pobreza que, además de estar expuestos a problemas de salud asociados a la obesidad, conllevan una desnutrición oculta, caracterizada por la falta de proteínas y micronutrientes como consecuencia de una alimentación monótona, basada en hidratos de car- bono. También, Temporelli y Viego (36) expresaron que la dieta de los hogares de bajos recursos está conformada por la canasta de alimentos a la que pueden acceder, que no necesariamente es compatible con sus deseos o recomendaciones nutricionales. A su vez, estos hallazgos confirman lo informado por la oms (37) según la cual entre 1980 y 2014, la prevalencia mundial de obesidad alcanzó valores por encima del doble de los registrados precedentemente.

A diferencia de lo observado en los restantes barrios, el Del Desembarco con NB I de o\% a 10\% no registró diferencias en las prevalencias de obesidad entre ambas cohortes y, además, presentó las más bajas encontradas en la C2. Autores tales como Marcus y Simkin (38) y Sanz Arazuri (39), postularon que el mayor nivel socio-económico se relaciona con el menor grado de sedentarismo. En este sentido, es probable que las familias presenten mejores condiciones que les permitan desarrollar más actividad física y/o controlar más equilibradamente la alimentación de los niños. Al respecto, Gracia-Arnaiz (40) señaló que en los últimos cincuenta años se han observado cuatro fenómenos distintos aunque estrechamente vinculados: el establecimiento del peso corporal ideal y las normas dietéticas; la construcción de la delgadez como un atributo de la salud y de la distinción social; el reconocimiento de la obesidad como enfermedad; y la transformación de la salud y el cuerpo en factores socioeconómicos y, por lo tanto, en oportunidades de negocio.

El estudio realizado permite concluir que, a pesar de que la mayoría de los barrios de Puerto Madryn conservan los niveles de pobreza estructural, el estado nutricional de los escolares varía. Mientras que la desnutrición crónica se mantiene o desciende, la obesidad aumenta distribuyéndose homogéneamente en todos los barrios. Esto confirma que la desnutrición está siendo reemplazada por la obesidad. Se considera que el análisis del estado nutricional complementado con la metodología de las NBI permite evaluar las transformaciones del contexto de residencia de una población, pudiendo identificarse los grupos vulnerables, que serían de interés en el diseño de política públicas en salud •

Agradecimientos: A Lorena Peralta y Vanesa Botteron (UNPSJB, Puerto Madryn), a Marcelo Gavirati y Delfina Palleres (IDEAus, CENPAT-CONICET) por su asistencia en los relevamientos antropométricos y a la traductora Adriana Di Maggio por la revisión idiomática.

Conflicto de Intereses: Ninguno.

Financiación: Se ha contado con el apoyo financiero del Consejo Nacional de Ciencia y Tecnología (CONICET) PIP2197, de la 
Agencia Nacional de Promoción Científica y Tecnológica (ANСут) PICT OC-AR 99-4-7391 y PICT OC-AR 1541 y 1145 , de la Universidad Nacional de La Plata (UNLP) 11/N 428 y de la Universidad Patagonia San Juan Bosco (UNSJB) SJB 10/C98.

\section{REFERENCIAS}

1. Salvia A. Sectores que ganan, sociedades que pierden: reestructuración y globalización en la Patagonia Austral. Estudios Sociológicos. 2001; 19(56): 439-66

2. Censo Nacional de población, hogares y vivienda. Instituto Nacional de Estadística y Censos (INDEC). 2010. Ministerio de Economía de la Nación. Buenos Aires. Argentina. [Internet] Disponible en: http://www. indec.gov.ar. Consultado en noviembre de 2016.

3. Sassone S, Gónzalez M, Matossian B. Ciudades patagónicas de la Argentina: atracción, crecimiento y diversidad migratoria. Aristas. (Mar del plata). 2011; 5: 109-30.

4. Kaminker SA. La dimensión racial en el análisis de la segregación residencial urbana en Puerto Madryn, Chubut. Pap trab. Cent Estud Interdiscip Etnolingüíst Antropol Sociocult. 2011; 22: 41-57.

5. Sassone S, Hughes J, Owen O. Análisis Geodemográfico de la Ciudad de Puerto Madryn. En: Sánchez, DC, director. Hacia un modelo de desarrollo turístico regional sustentable: el crecimiento de Puerto Madryn y el vaciamiento de la meseta contigua. Buenos Aires, CONICET-IMHICIHU; 2015

6. Sassone SM, Hughes JC, Owen OM. Nuevos asentamientos precarios en la periferia de Puerto Madryn: Desafío para las intervenciones urbanísticas. Sociedad Argentina de Estudios Geográficos. Boletín No 132 Especial; Actas Congreso Internacional de Geografía. $74^{\circ}$ Semana de Geografía Trevelin; 2013.

7. Dirección General de Estadística y Censos (DGEyC). [Internet]. Disponible en: https://bit.ly/2GzluNF. Consultado en enero de 2017.

8. Feres JC, Mancero X. El método de las necesidades básicas insatisfechas (NBI) y sus aplicaciones en América Latina. Serie de estudios estadísticos y prospectivos N ${ }^{\circ} 7$ Santiago de Chile, Cepal; 2001.

9. Ibarbia M. La pobreza según las Necesidades Básicas Insatisfechas. En: Chubut Perfiles 5. La pobreza en Chubut. Dirección General de Estadística y Censos (DGEyC). Centro de Documentación y Difusión. Chubut; DGEyC; 2014.

10. Dirección Nacional de Relaciones Económicas con las Provincias (DINREP). Subsecretaría de Relaciones con Provincias. Ministerio de Economía y Finanzas Públicas de la Nación. Necesidades Básicas Insatisfechas (NBI) 2014. [Internet] Disponible en: https://bit.ly/1JX4pHm.

11. Beccaría LA, Feres JC, Sáinz P. Medición de la pobreza: situación actual de los conceptos y métodos. Informe del Seminario de Santiago, 7-9 de mayo de 1997. En: Cuarto Taller Regional sobre la Medición de la Pobreza: el Método de las Líneas de Pobreza: documentos presentados-LC/R. 1985-2000. Buenos Aires; 2000.

12. Instituto Nacional de Estadísticas y Censos (INDEC). La pobreza en la Argentina. Indicadores de Necesidades Básicas Insatisfechas a partir de los datos del Censo Nacional de Población y Vivienda 1980. Buenos Aires: Estudios INDEC; 1984.

13. Montilva M, Ferrer MA, Nieto R, Ontiveros Y, Durán L, Mendoza MA. Uso del método: necesidades básicas insatisfechas en la detección de comunidades con riesgo de desnutrición. An Venez Nutr. (Caracas. 2003; 16(1): 16-22.

14. Tanner JM. A history of the study of human growth. Cambridge: Cambridge University Press; 1981.

15. Fogel RW. Physical growth as a measure of the economic wellbeing of populations: the eighteenth and nineteenth centuries. In: Falkner F, Tanner JM, eds. Human growth. A comprehensive treatise. New York, NY: Plenum; 1986.

16. World Health Organization Expert Committee. Physical Status: The Use and Interpretation of Anthropometry, WHO Tech Rep Ser N 854. Geneva World Health Organization; 1995.
17. Ulijaszek S, Komlos J. From a History of Anthropometry to Anthropometric History. In: Mascie-Taylor CGN, Yasukouchi A, Ulijaszek S, eds. Human variation: from the laboratory to the field. Boca Raton, FL: CRC Press; 2010.

18. Juiz de Trogliero C, Morasso MDC. Obesidad y nivel socioeconómico en escolares y adolescentes de la ciudad de Salta. Arch Argent Pediatr. 2002; 100(5): 360-66.

19. Poletti C, Oscar H, Lilian Barrios M. Sobrepeso y obesidad como componentes de la malnutrición, en escolares de la ciudad de Corrientes: Argentina. Rev Chil Ped. (Santiago). 2003; 74(5): 499-503.

20. Tazza R, Bullón L. ¿Obesidad o desnutrición?: Problema actual de los niños peruanos menores de 5 años. An Fac Med. 2006; 67(3):214-23.

21. Mispireta ML, Rosas ÁM, Velásquez JE, Lescano AG, Lanata CF. Transición nutricional en el Perú, 1991-2005. Rev Peru Med Exp Salud Pública. 2007; 24(2): 129-35.

22. Lohman TG, Roche AF, Martorell R. Anthropometric standarization reference manual. Illinois: Human Kinetics Books; 1988.

23. Dahinten SL, Zavatti JR. Transición nutricional en Patagonia. Rev Arg Antrop Biol. 2003; 5(1): 64.

24. Botterón TV, Zavatti JR, Giménez D, Gallastegui MS, Dahinten SL. Influencia del nivel socioeconómico sobre el dimorfismo sexual de escolares patagónicos. Rev Arg Antrop Biol. 2003; 5(1):119.

25. Arakaki A. Cuatro décadas de Necesidades Básicas Insatisfechas en Argentina. Trab soc. 2016; 27: 269-90.

26. Pickett KE, Pearl M. Multilevel analyses of neighbourhood socioeconomic context and health outcomes: a critical review. J Epidemiol Community Health. 2001; 55(2): 111-22.

27. Diez Roux AV, Mair C. Neighborhoods and health. Ann N Y Acad Sci. 2010; 1 186: 125-45.

28. Kawachi I, Berkman LF. Introduction. In: Kawachi I, Berkman LF (Eds). Neighborhoods and health. New York: Oxford University Press; 2003.

29. Papas MA, Alberg AJ, Ewing R, Helzlsouer KJ, Gary TL, Klassen AC. The built environment and obesity. Epidemiol Rev. 2007; 29(1): 129-43.

30. Koplan JP, Liverman CT, Kraak VA,eds. Preventing childhood obesity: health in the balance. Washington: The National Academies Press; 2005.

31. Singh GK, Kogan MD, van Dyck PC. A multilevel analysis of state and regional disparities in childhood and adolescent obesity in the United States. J Community Health. 2008; 33(2): 90-102.

32. Kaminker SA, Velásquez RY. Programa de mejoramiento de barrios en la patagonia central: regularización de la informalidad urbana en Puerto Madryn, Chubut. Cuad urbano. 2015; 18(18): 89-110.

33. Pedraza DF. Obesidad y Pobreza: marco conceptual para su análisis en latinoamérica. Saúde Soc. São Paulo. 2009; 18(1): 103-17.

34. Sassone SM, Hughes JC, Owen OM, Sánchez D, Llanos E, Barrios L, Bayón S, Lorenzi N. Apropiación territorial y reproducción cultural de migrantes en contextos urbanos: Trelew y Puerto Madryn. En: Monti A, Alcarraz G, Ferrari MP, coord. Miradas Geográficas de la Patagonia. Encuentros con la investigación y la docencia. Trelew: Biblioteca Popular Agustín Álvarez; 2012.

35. Aguirre P. Estrategias de consumo: qué comen los argentinos que comen. Editorial CIEPP, Buenos Aires; 2006.

36. Temporelli KL, Viego VN. Condicionantes socioeconómicos y obesidad en adultos: evidencia basada en regresiones por cuantiles y datos de panel. Rev. salud pública (Bogotá). 2016; 18(4): 516-29.

37. Organización Mundial de la Salud. Obesidad y sobrepeso. Nota descriptiva $N^{\circ} 311$ Junio de 2016. [Internet] Disponible en: https://bit.ly/1hBxlq3. Consultado: Enero 2017.

38. Marcus BH, Simkin LR. The Transtheoretical Model: Applications to exercise behavior. Med Sci Sports Exerc. 1994; 26(11):1400-04.

39. Sanz Arazuri E. La práctica físico-deportiva de tiempo libre en universitarios: Análisis y propuestas de mejora. Logroño: Universidad de La Rioja; 2005.

40. Gracia-Arnaiz, M. Fat bodies and thin bodies. Cultural, biomedical and market discourses on obesity. Appetite. 2010; 55(2): 119-25. 